\title{
Castleman disease and TAFRO syndrome
}

\author{
Yasufumi Masaki $^{1}$ (D) Kotaro Arita ${ }^{1} \cdot$ Tomoyuki Sakai $^{1} \cdot$ Kazue Takai $^{2} \cdot$ Sadao Aoki $^{3} \cdot$ Hiroshi Kawabata $^{4}$
}

Received: 9 October 2021 / Accepted: 11 January 2022 / Published online: 19 January 2022

(c) The Author(s), under exclusive licence to Springer-Verlag GmbH Germany, part of Springer Nature 2022

\begin{abstract}
Although Castleman disease was first described in 1956, this disease includes various conditions, including unicentric Castleman disease with hyaline vascular histology, human herpesvirus-8 (HHV-8) related multicentric Castleman disease, idiopathic multicentric Castleman disease, and mimics of Castleman disease associated with other conditions. To date, Castleman disease remains incompletely understood due to its rareness and difficulties in clinical and pathological diagnosis. TAFRO syndrome was reported in Japan in 2010. Because lymph node histology is similar in patients with TAFRO syndrome and Castleman disease, TAFRO syndrome is described as a related disorder of Castleman disease. Clinically, however, these conditions differ markedly. Although elevated interleukin-6 (IL-6) expression is characteristic of Castleman disease, increased expression of IL-6 may occur in patients with other diseases, making elevated IL-6 unsuitable for differential diagnosis. Further understanding of these disorders requires the identification of novel disease-specific biomarkers. This review article therefore outlines the characteristics of Castleman disease and TAFRO syndrome.
\end{abstract}

Keywords IL- $6 \cdot$ HHV-8 $\cdot$ POEMS syndrome $\cdot$ Anasarca $\cdot$ Thrombocytopenia $\cdot$ Cytokine storm

\section{What is Castleman disease?}

In 1956, Benjamin Castleman described patients with mediastinal tumors mimicking thymomas, accompanied by lymphoid follicle hyperplasia and hyalinized vessels penetrating into the germinal centers [1]. This condition, named Castleman disease (CD), has also been called giant lymph node hyperplasia, lymphnodal hamartoma, lymph node hamartoma, follicular lympho-reticuloma, benign giant lymphoma, angiofollicular lymph node hyperplasia, and angiomatous lymphoid hamartoma.

Yasufumi Masaki

yasum@kanazawa-med.ac.jp

1 Department of Hematology and Immunology, Kanazawa Medical University, 1-1 Daigaku, Uchinada, Kahoku-gun, Ishikawa 920-0293, Japan

2 Department of Hematology, Niigata City General Hospital, Niigata, Japan

3 Department of Pathophysiology, Faculty of Pharmaceutical Sciences, Niigata University of Pharmacy and Applied Life Sciences, Niigata, Japan

4 Department of Hematology, National Hospital Organization Kyoto Medical Center, Kyoto, Japan
$\mathrm{CD}$ has been classified clinically as unicentric (localized, unicentric Castleman disease [UCD]) and multicentric (systemic, multicentric Castleman disease [MCD]). Histopathologically, CD has been classified as hyaline vascular (HV) type, plasma cell (PC) type, mixed type, hyper-vascular type, and plasmablastic type [2]. Most patients with UCD have HV histology, with the remainder having PC histology. MCD has been etiologically classified by the Castleman Disease Collaborative Network (CDCN) as human herpesvirus 8 (HHV-8)-related; HHV-8-unrelated, also called idiopathic MCD (iMCD); POEMS(polyneuropathy, organomegaly, endocrinopathy, M-protein, and skin changes) syndrome-related; and others [2]. The latter two classifications, however, may be categorized together (Fig. 1). The histopathological characteristics of Japanese patients with MCD have been described in detail [3].

Because patients with UCD and HV histology do not demonstrate hyper-cytokine syndrome and are curable by surgical resection, this disease is considered a distinct disease entity [4-6].

MCD is characterized by the overexpression of interleukin-6 (IL-6), or hyper-IL-6 syndrome, with polyclonal lymphadenopathy. MCD in Western countries is frequently HHV-8-related, with viral IL-6 expression causing various symptoms [7]. The progression of B cell differentiation in 


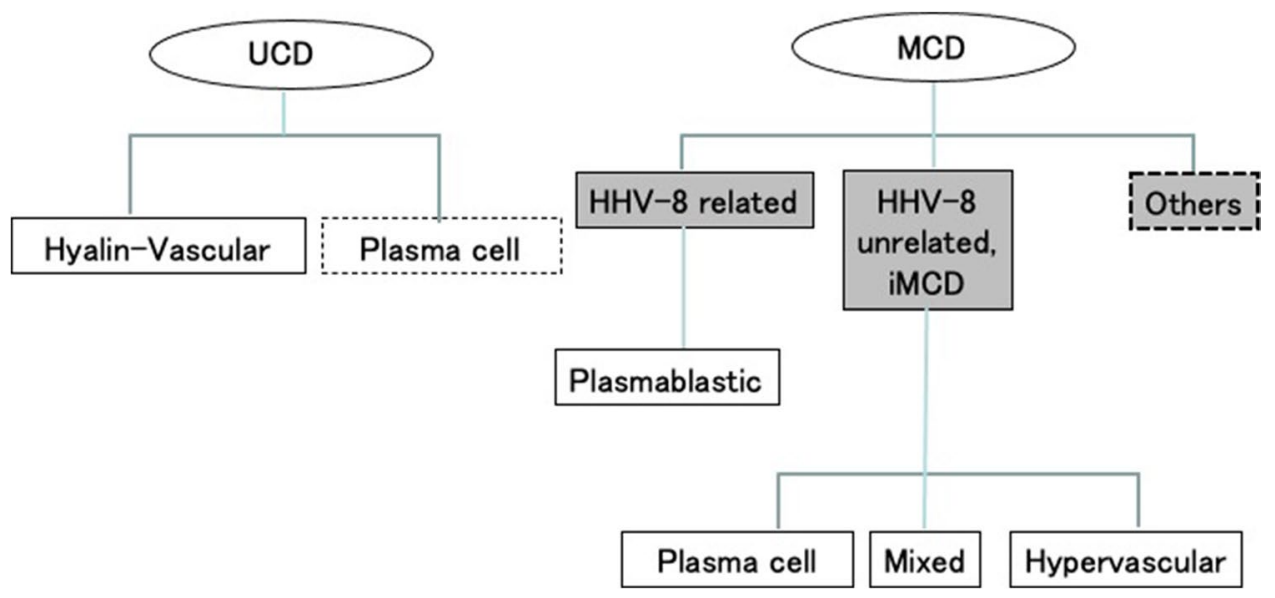

Fig. 1 Classification of types of Castleman disease. Castleman disease is clinically classified as unicentric Castleman disease (UCD) and multicentric Castleman disease (MCD) and was histopathologically classified as hyaline vascular, plasma cell, mixed, hyper-vascular, and plasmablastic types. Most patients with UCD have hyaline vascular histology, with the remainder having plasma cell histology.

these patients induces plasma cell expansion and polyclonal hypergammopathy. Moreover, the induction of vascular endothelial growth factor (VEGF) production causes angiogenesis, and differentiation of megakaryocytes in bone marrow causes thrombocytosis. Furthermore, the increased production in the liver of acute inflammatory proteins, such as C-reactive protein (CRP), fibrinogen, and serum amyloid A (SAA), as well as hepcidin, inhibits absorption of iron in the gastrointestinal tract and inhibits iron-recycling in the reticuloendothelial systems, resulting in normo- to microcytic anemia. A retrospective analysis of patients with MCD showed that more than $50 \%$ of these patients had iMCD, even in the USA [2]. Most patients with MCD in Japan have iMCD, with the annual incidence rate of MCD in Japan estimated at 309-731, equal to 2.4-5.8 per million individuals [8]. MCD, with or without HHV-8, is characterized by hyper-IL-6 syndrome, resulting in systemic symptoms such as polyclonal hypergammopathy, fever, hyper-inflammation, anemia, and thrombocytosis. Initially, patients with MCD were treated with glucocorticoids, but their efficacy was restricted. The monoclonal anti-IL-6 receptor antibody tocilizumab has been approved by the National Health Insurance in Japan, whereas the monoclonal anti-IL-6 antibody siltuximab and the monoclonal anti-CD20 antibody rituximab have been approved for treatment in the USA (Table 1) [9].

\section{What is TAFRO syndrome?}

TAFRO syndrome, first described in 2010 , is a condition characterized by thrombocytopenia, anasarca (edema, pleural effusion, and ascites), fever, reticulin myelofibrosis (or
MCD is etiologically classified as human herpesvirus 8 (HHV-8)-related, HHV-8-unrelated (idiopathic MCD [iMCD]), and other, including POEMS syndrome, TAFRO syndrome, IgG4-related disease, malignancies (lymphoma, cancer, and sarcoma), autoimmune diseases (systemic lupus erythematosus, Sjögren's syndrome, and vasculitis), and infections (e.g., tuberculosis)

renal insufficiency), and organomegaly (hepatosplenomegaly and lymphadenopathy) [11]. The annual incidence of TAFRO syndrome in Japan was estimated to be 110-502, equivalent to $0.9-4.9$ per million individuals [8]. Although its etiology is unknown, TAFRO syndrome is a hyper-inflammation syndrome associated with marked thrombocytopenia and anasarca. The lymph node histopathology of TAFRO syndrome mimics that of MCD; thus, TAFRO syndrome is thought to be a variant of iMCD (iMCD-TAFRO) for some researchers [12-14]. However, lymph node biopsies may be difficult or impossible to obtain from patients with TAFRO syndrome due to anasarca, bleeding tendency, and/or the smallness or unclearness of the target lymph node. Therefore, Japanese diagnostic criteria for TAFRO syndrome have included patients without histological confirmation of lymph nodes $[10,15]$.

To identify factors prognostic for TAFRO syndrome, data of 83 patients with TAFRO syndrome were obtained from the registry in the Multicenter Collaborative Retrospective Study for Establishing the Concept of TAFRO Syndrome in Japan. Univariable analysis identified several potential prognostic factors, with a multivariable Cox proportional hazards model finding that age $\geq 60$ years and D-dimer concentration $\geq 18 \mu \mathrm{g} / \mathrm{dL}$ remain significant predictors of poor overall survival. This led to the development of a prognostic scoring system for TAFRO syndrome (TS-PSS), with patients in the study cohort stratified into those at low, intermediate, and high risk for TAFRO syndrome. Further studies in independent patient cohorts are needed to verify the prognostic accuracy of the TS-PSS [16].

Although mortality rates are high in patients with TAFRO syndrome, a single optimal treatment strategy has not yet 
Table 1 Treatment strategies for patients with iMCD and TAFRO syndrome

iMCD (ref [9])

1. For non-severe patients

Siltuximab (monoclonal anti-IL-6 antibody) $+/$ - Steroids

Tocilizumab (monoclonal anti-IL-6 receptor antibody) $+/$ - Steroids

Rituximab (monoclonal anti-CD20 antibody) +/- Steroids

2. For severe patients

Siltuximab +high dose Steroids

Tocilizumab +high dose Steroids

TAFRO syndrome (ref [10])

1. First line treatment

Glucocorticoid, high dose $(1 \mathrm{mg} / \mathrm{kg}$ per daily dose of prednisolone or pulse therapy using $500-1,000 \mathrm{mg}$ daily dose of methylprednisolone)

2. Second line treatment

Rituximab

Tocilizumab

Cyclosporin A

Thrombopoietin receptor agonists romiplostim and eltrombopag: for patients with persistent thrombocytopenia.

Other options

Plasma exchange, high dose cyclophosphamide, thalidomide, lenalidomide, bortezomib rapamycin and combination chemotherapy such as

CHOP (cyclophosphamide, doxorubicin, vincristine, and prednisolone) have been successful in the treatment of selected patients.

Splenectomy and high-dose gamma-globulin have not been shown effective.

been established. A list of agents that have been used in various cases is shown in Table 1 . In our retrospective study, 68 patients received first-line corticosteroid therapy, with second-line treatment consisting of tocilizumab (Toc) in 21 patients, cyclosporine $\mathrm{A}(\mathrm{CsA})$ in 14, and rituximab (Rit) in eight, in addition to corticosteroids. The primary study endpoint was time to next treatment or death (TTNT). Kaplan-Meier analysis showed that the median TTNT in the Toc, CsA, and Rit groups were 3.0 months, 11.5 months, and not reached, respectively. TTNT tended to be longer in the Rit than in the Toc group. In contrast, there were no significant differences in overall survival among the three groups, indicating that subsequent salvage therapies rescued a large proportion of patients who failed second-line treatments [17]. Further studies are warranted to establish the optimal treatment strategies for patients with this syndrome.

\section{Differences between MCD and TAFRO syndrome}

Clinical data were collected from more than 220 patients with iMCD, TAFRO syndrome, and other mimickers, and their differences were analyzed [18]. Various clinical symptoms differed in patients with TAFRO syndrome and typical iMCD, indicating that the former is a distinct clinical entity. In most patients, iMCD has an indolent and chronic clinical course, accompanied by polyclonal hypergammopathy, multiple lymphadenopathy, hyper-inflammation, anemia, and thrombocytosis. In contrast, the onset and course of TAFRO syndrome is acute, with rapid deterioration and serious disease. Moreover, most patients with TAFRO syndrome were found to have normo- or hypo-gammaglobulinemia, thrombocytopenia, small or unclear lymphadenopathy, and anasarca including massive pleural effusion and ascites. Lymph node biopsies cannot be obtained from some patients with TAFRO syndrome because of anasarca, bleeding tendency due to thrombocytopenia, and small or unclear lymphadenopathy. However, lymph node histology is similar, with overlapping characteristics, in patients with TAFRO syndrome and iMCD. To differentiate among the clinical features of TAFRO syndrome with and without iMCD histopathology and iMCD-not otherwise specified (NOS), 220 patients with these conditions were retrospectively analyzed. Rates of fever, anasarca, renal failure, and mortality were significantly higher in patients with TAFRO syndrome with and without iMCD than in patients with iMCD-NOS. Laboratory findings showed that blood ureanitrogen, creatinine, CRP, ALP, $\gamma$-GTP, D dimer, and FDP concentrations were significantly higher in patients with TAFRO syndrome with and without iMCD than in patients with iMCD-NOS, whereas platelet counts and total protein and albumin concentrations were significantly lower in patients with TAFRO syndrome with and without iMCD than in patients with iMCD-NOS. In contrast, serum IgG, $\operatorname{IgA}$, and $\operatorname{IgM}$ concentrations were significantly higher in patients with iMCD-NOS than in patients with TAFRO syndrome with and without iMCD. Kaplan-Meier analyses revealed that the overall survival (OS) rates of patients with TAFRO syndrome decreased rapidly within 24 months of diagnosis, by which time one-third of the patients had died. OS rates, however, did not differ significantly between 
patients with TAFRO with and without iMCD. In contrast, more than $90 \%$ of patients with iMCD-NOS were still alive 10 years after diagnosis. Taken together, these findings indicate that TAFRO syndrome with and without iMCD should be considered the same entity, requiring prompt diagnosis and intensive care [18].

\section{Cytokine storm and hyper-IL-6 syndrome (Fig. 2)}

Because the diagnosis of $\mathrm{CD}$ depends on histological findings, hyper-inflammation, and polyclonal lymphoproliferation conditions, patients with suspicion of malignant lymphoma without proven monoclonality can be diagnosed with MCD. Lymphadenopathy may also be present in patients with TAFRO syndrome, POEMS syndrome [19], and IgG4-related disease [20]. Lymph node biopsy of these patients may result in a histologic diagnosis of MCD or a condition undistinguishable from PC-, mixed-, or hyper-vascular-type MCD. Fever is present in patients with both TAFRO syndrome and POEMS syndrome, making these conditions important in the differential diagnosis of fever of unknown origin. In contrast, IgG4-related disease is not characterized by high fever or markedly elevated CRP. Furthermore, patients with lymphomas, such as angioimmunoblastic T cell lymphoma and intravascular large B cell lymphoma [21], accompanied by hyper-cytokinemia and generalized inflammation, may have MCD-like clinical symptoms and satellite lymph node biopsies may yield MCD-like histopathological findings. Symptoms and histopathology may be similar in patients with cytokine-producing cancers and sarcomas [22]; various collagen vascular diseases, such as systemic lupus erythematosus, Sjögren's syndrome [23], and vasculitis syndrome; and various infections accompanied by hyper-inflammation. Careful diagnosis of iMCD requires the differential diagnoses of these conditions.

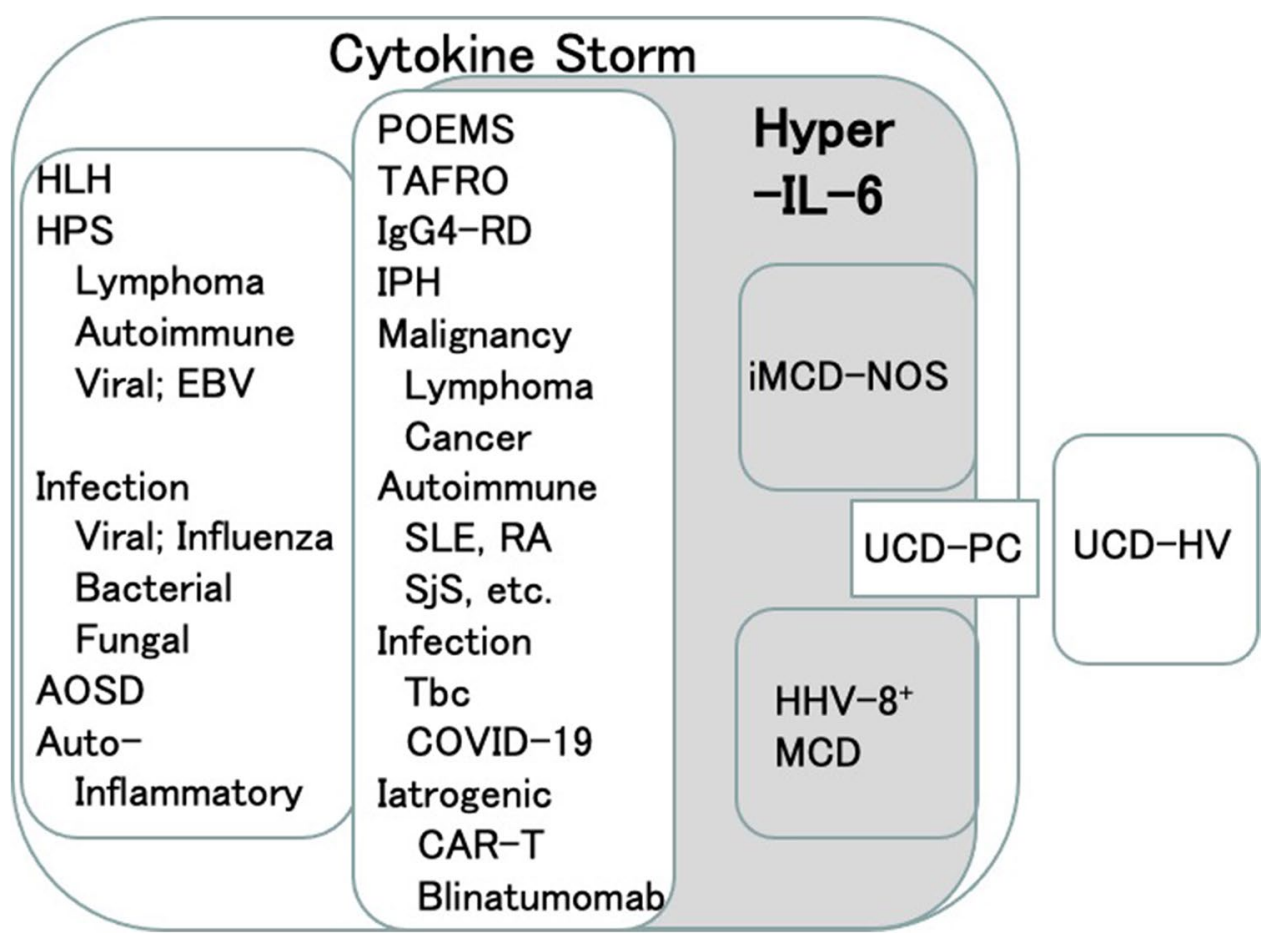

Fig. 2 Cytokine storm and hyper-IL-6 syndrome. Hyper-IL-6 syndrome occurs frequently in patients with HHV8-related MCD and iMCD-NOS and in PC-type UCD. Clinical symptoms and lymph node histology due to hyper-IL6 syndrome have also been observed in patients with POEMS syndrome; TAFRO syndrome; idiopathic portal hypertension (IPH); various malignancies, such as lymphoma and cancer; various autoimmune diseases, such as systemic lupus erythematosus (SLE), rheumatoid arthritis (RA), and Sjögren' s syndrome $(\mathrm{SjS})$; some infections, such as with tuberculosis $(\mathrm{Tbc})$ and
COVID-19; and some iatrogenic conditions, such as chimeric antigen receptor T-cell (CAR-T) therapy and treatment with blinatumomab. Although some patients with IgG4-related disease (IgG4-RD) demonstrate hyper-IL-6 syndrome, most do not. Other than hyper-IL-6 syndrome, hemophagocytic lymphohistiocytosis (HLH), hemophagocytic syndrome (HPS), some severe infections, adult-onset Still disease, and auto-inflammatory syndrome causes hyper-cytokine storm. In contrast, HV-type UCD is not associated with cytokine storm 


\section{Future prospects}

Although elevated IL-6 expression is characteristic of CD, increased expression of IL-6 may occur in patients with other diseases, making elevated IL-6 unsuitable for differential diagnosis. Further understanding of these disorders requires the identification of novel disease-specific biomarkers.

Acknowledgements We thank all participants in the All Japan, Ministry of Health, Labor, and Welfare (MHLW) TAFRO syndrome team and Castleman disease team for their help and critical discussion. We would like to thank David Price (http://www.english-for-scientists.com) for English language editing.

Author contribution YM prepared and wrote the manuscript. KA, TS, $\mathrm{KT}$, SA, and HK mainly enrolled cases in the retrospective clinical study and discussed and analyzed data. All authors read and approved the final manuscript.

Funding This study was supported in part by grants from the Ministry of Health, Labor and Welfare, Japan (H29 Nanchi, etc. (Nan)General-019, H27-28 Nanchi, etc. (Nan)-General-002 and -008) and by the Japanese Ministry of Education, Culture, Sports, Science and Technology (Grant No.17591060 and 15K09510), the Kanazawa Medical University Research Foundation (Grant Nos. S2004-16 and S2007-5), Grant for Assist KAKEN from Kanazawa Medical University (Grant No.K2011-7), Grant for Project Research from High-Tech Research Center of Kanazawa Medical University (Grant No.H201111), and Grant for Alumni Research(A) from Kanazawa Medical University(AR2012-06).

Availability of data and material Not applicable.

Code availability Not applicable.

\section{Declarations}

Ethics approval Not applicable.

Consent to participate Not applicable.

Consent for publication Not applicable.

Conflict of interest YM has received research grants from Kyowa Hakko Kirin Pharma, Astellas Pharma, Eisai Pharma, Ono Pharma, Pfizer Pharma, Asahi Kasei Pharma, MSD Pharma, Daiichi-Sankyo Pharma, Taisho Pharma, Taiho Pharma, Takeda Pharma, Chugai Pharma, Teijin Pharma, Nippon Kayaku, and Mochida Pharma, outside the submitted work. All other authors declare no competing interests.

\section{References}

1. Castleman B, Iverson L, Menendez VP (1956) Localized mediastinal lymph node hyperplasia resembling lymphoma. Cancer 9:822-830. https://doi.org/10.1002/1097-0142(195607/08)9:4\% 3c822::aid-cncr2820090430\%3e3.0.co;2-4
2. Fajgenbaum DC, Uldrick TS, Bagg A, Frank D, Wu D, Srkalovic G, Simpson D, Liu AY, Menke D, Chandrakasan S, Lechowicz MJ, Wong RS, Pierson S, Paessler M, Rossi JF, Ide M, Ruth J, Croglio M, Suarez A, Krymskaya V, Chadburn A, Colleoni G, Nasta S, Jayanthan R, Nabel CS, Casper C, Dispenzieri A, Fosså A, Kelleher D, Kurzrock R, Voorhees P, Dogan A, Yoshizaki K, van Rhee F, Oksenhendler E, Jaffe ES, Elenitoba-Johnson KS, Lim MS (2017) International, evidence-based consensus diagnostic criteria for HHV-8-negative/idiopathic multicentric Castleman disease. Blood 129:1646-1657. https://doi.org/10. 1182/blood-2016-10-746933

3. Kurose N, Futatsuya C, Mizutani KI, Kumagai M, Shioya A, Guo X, Aikawa A, Nakada S, Fujimoto S, Kawabata H, Masaki Y, Takai K, Aoki S, Kojima M, Nakamura S, Yamada S (2018) The clinicopathological comparison among nodal cases of idiopathic multicentric Castleman disease with and without TAFRO syndrome. Hum Pathol 77:130-138. https://doi.org/10.1016/j. humpath.2018.04.001

4. Ye B, Gao SG, Li W, Yang LH, Zhao SH, Ma K, Zhu XL, Liu XY, Sun KLA (2010) retrospective study of unicentric and multicentric Castleman's disease: a report of 52 patients. Med Oncol 27:1171-1178. https://doi.org/10.1007/s12032-009-9355-0

5. Talat N, Belgaumkar AP, Schulte KM (2012) Surgery in Castleman's disease: a systematic review of 404 published cases. Ann Surg 255:677-684. https://doi.org/10.1097/SLA.0b013e3182 $49 \mathrm{dcdc}$

6. Jiang JP, Shen XF, Du JF, Guan WX (2018) A retrospective study of 34 patients with unicentric and multicentric Castleman's disease: experience from a single institution. Oncol Lett 15:2407-2412. https://doi.org/10.3892/ol.2017.7625

7. Yu L, Tu M, Cortes J, Xu-Monette ZY, Miranda RN, Zhang J, Orlowski RZ, Neelapu S, Boddu PC, Akosile MA, Uldrick TS, Yarchoan R, Medeiros LJ, Li Y, Fajgenbaum DC, Young KH (2017) Clinical and pathological characteristics of HIV- and HHV-8-negative Castleman disease. Blood 129:1658-1668. https://doi.org/10.1182/blood-2016-11-748855

8. Masaki Y, Kawabata H, Fujimoto S, Kawano M, Iwaki N, Kotani T, Nakashima A, Kurose N, Takai K, Suzuki R, Aoki S (2019) Epidemiological analysis of multicentric and unicentric Castleman disease and TAFRO syndrome in Japan. J Clin Exp Hematop 59:175-178. https://doi.org/10.3960/jslrt.19021

9. van Rhee F, Voorhees P, Dispenzieri A, Fosså A, Srkalovic G, Ide M, Munshi N, Schey S, Streetly M, Pierson SK, Partridge HL, Mukherjee S, Shilling D, Stone K, Greenway A, Ruth J, Lechowicz MJ, Chandrakasan S, Jayanthan R, Jaffe ES, Leitch H, Pemmaraju N, Chadburn A, Lim MS, Elenitoba-Johnson KS, Krymskaya V, Goodman A, Hoffmann C, Zinzani PL, Ferrero S, Terriou L, Sato Y, Simpson D, Wong R, Rossi JF, Nasta S, Yoshizaki K, Kurzrock R, Uldrick TS, Casper C, Oksenhendler E, Fajgenbaum DC (2019) International, evidence-based consensus treatment guidelines for idiopathic multicentric Castleman disease. Blood 132:2115-2124. https://doi.org/10.1182/ blood-2018-07-862334

10. Masaki Y, Kawabata H, Takai K, Kojima M, Tsukamoto N, Ishigaki Y, Kurose N, Ide M, Murakami J, Nara K, Yamamoto H, Ozawa Y, Takahashi H, Miura K, Miyauchi T, Yoshida S, Momoi A, Awano N, Ikushima S, Ohta Y, Furuta N, Fujimoto S, Kawanami H, Sakai T, Kawanami T, Fujita Y, Fukushima T, Nakamura S, Kinoshita T, Aoki S (2016) Proposed diagnostic criteria, disease severity classification and treatment strategy for TAFRO syndrome, 2015 version. Int J Hematol 103:686-692. https://doi. org/10.1007/s12185-016-1979-1

11. Takai K, Nikkuni K, Shibuya H, Hashidate H (2010) Thrombocytopenia with mild bone marrow fibrosis accompanied by fever, pleural effusion, ascites and hepatosplenomegaly. Rinsho Ketsueki 51:320-325 (in Japanese) 
12. Iwaki N, Fajgenbaum DC, Nabel CS, Gion Y, Kondo E, Kawano M, Masunari T, Yoshida I, Moro H, Nikkuni K, Takai K, Matsue K, Kurosawa M, Hagihara M, Saito A, Okamoto M, Yokota K, Hiraiwa S, Nakamura N, Nakao S, Yoshino T, Sato Y (2016) Clinicopathologic analysis of TAFRO syndrome demonstrates a distinct subtype of HHV-8-negative multicentric Castleman disease. Am J Hematol 91:220-226. https://doi.org/10.1002/ajh.24242

13. Nishimura Y, Fajgenbaum DC, Pierson SK, Iwaki N, Nishikori A, Kawano M, Nakamura N, Izutsu K, Takeuchi K, Nishimura MF, Maeda Y, Otsuka F, Yoshizaki K, Oksenhendler E, van Rhee F, Sato Y (2021) Validated International Definition of the TAFRO clinical subtype of idiopathic multicentric Castleman disease. Am J Hematol. https://doi.org/10.1002/ajh.26292

14. Dispenzieri A, Fajgenbaum DC (2020) Overview of Castleman disease. Blood 135:1353-1364. https://doi.org/10.1182/blood. 2019000931

15. Masaki Y, Kawabata H, Takai K, Tsukamoto N, Fujimoto S, Ishigaki Y, Kurose N, Miura K, Nakamura S, Aoki S, Japanese TAFRO Syndrome Research Team (2020) 2019 Updated diagnostic criteria and disease severity classification for TAFRO syndrome. Int J Hematol 111:155-158. https://doi.org/10.1007/ s12185-019-02780-1

16. Kawabata H, Fujimoto S, Sakai T, Yanagisawa H, Kitawaki T, Nara K, Hagihara M, Yamamoto H, Tanimizu M, Kato C, Origuchi T, Sunami K, Sunami Y, Masunari T, Nakamura N, Kobayashi M, Yamagami K, Miura K, Takai K, Aoki S, Tsukamoto N, Masaki Y (2021) Patient's age and D-dimer levels predict the prognosis in patients with TAFRO syndrome. Int $\mathrm{J}$ Hematol 114:179-188. https://doi.org/10.1007/s12185-021-03159-x

17. Fujimoto S, Kawabata H, Sakai T, Yanagisawa H, Nishikori M, Nara K, Ohara S, Tsukamoto N, Kurose N, Yamada S, Takai K, Aoki S, Masaki Y (2021) Optimal treatments for TAFRO syndrome: a retrospective surveillance study in Japan. Int J Hematol 113:73-80. https://doi.org/10.1007/s12185-020-03008-3

18. Fujimoto S, Sakai T, Kawabata H, Kurose N, Yamada S, Takai K, Aoki S, Kuroda J, Ide M, Setoguchi K, Tsukamoto N,
Iwao-Kawanami H, Kawanami T, Mizuta S, Fukushima T, Masaki Y (2019) Is TAFRO syndrome a subtype of idiopathic multicentric Castleman disease? Am J Hematol 94:975-983. https://doi. org/10.1002/ajh.25554

19. Suichi T, Misawa S, Sekiguchi Y, Shibuya K, Tsuneyama A, Suzuki YI, Nakamura K, Kano H, Kuwabara S (2020) Treatment response and prognosis of POEMS syndrome coexisting with Castleman disease. J Neurol Sci 413:116771. https://doi.org/10. 1016/j.jns.2020.116771

20. Sato Y, Notohara K, Kojima M, Takata K, Masaki Y, Yoshino T (2010) IgG4-related disease: historical overview and pathology of hematological disorders. Pathol Int 60:247-258. https://doi.org/ 10.1111/j.1440-1827.2010.02524.x

21. Shiroshita K, Kikuchi T, Okayama M, Kasahara H, Kamiya T, Shimizu T, Kurose N, Masaki Y, Okamoto S (2020) Interleukin6-producing intravascular large B-cell lymphoma with lymphadenopathy mimicking the histology of multicentric Castleman disease. Intern Med 59:3061-3065. https://doi.org/10.2169/inter nalmedicine.5046-20

22. Fujimoto S, Sakai T, Kawabata H, Kurose N, Yamada S, Doai M, Matoba M, Iwao-Kawanami H, Kawanami T, Mizuta S, Fukushima T, Mizumaki Y, Yamaguchi M, Masaki Y (2019) Osteosarcoma manifesting systemic inflammation and histological features mimicking plasma cell type Castleman disease. Intern Med 58:2555-2560. https://doi.org/10.2169/internalmedicine.2738-19

23. Fujimoto S, Kawabata H, Kurose N, Kawanami-Iwao H, Sakai T, Kawanami T, Fujita Y, Fukushima T, Masaki Y (2017) Sjögren's syndrome manifesting as clinicopathological features of TAFRO syndrome: a case report. Medicine 96:e9220. https://doi.org/10. 1097/MD.0000000000009220

Publisher's note Springer Nature remains neutral with regard to jurisdictional claims in published maps and institutional affiliations. 